\title{
A Series of Metal-Organic Frameworks: Syntheses, Structures and Luminescent Detection, Gas Adsorption, Magnetic Properties
}

\author{
Shui-Sheng Chen, ${ }^{*},+*$ Shuai-Shuai Han, ${ }^{\dagger}$ Cheng-Bing Ma, ${ }^{*}, \dagger$ Wei-Dong Li, ${ }^{\dagger}$ \\ and Yue Zhao*,*
}

$\dagger$ School of Chemistry and Chemical Engineering, Fuyang Normal University, Fuyang 236041, China

$\ddagger$ Coordination Chemistry Institute, State Key Laboratory of Coordination Chemistry, Nanjing University, Nanjing 210093, China

Table S1 Selected bond lengths $[\AA]$ and bond angles $\left[^{\circ}\right]$ for MOFs 1 - 6

$\begin{array}{llll}1 & & & \\ \mathrm{Cd}(1)-\mathrm{O}(1) & 2.372(2) & \mathrm{Cd}(1)-\mathrm{O}(2) & 2.391(2) \\ \mathrm{Cd}(1)-\mathrm{O}(5) & 2.293(19) & \mathrm{Cd}(1)-\mathrm{O}(6) \# 1 & 2.302(2) \\ \mathrm{Cd}(1)-\mathrm{N}(1) & 2.264(2) & \mathrm{Cd}(1)-\mathrm{N}(5) \# 3 & 2.287(2) \\ \mathrm{Cd}(2)-\mathrm{O}(3) \# 1 & 2.323(2) & \mathrm{Cd}(2)-\mathrm{O}(4) \# 3 & 2.281(2) \\ \mathrm{Cd}(2)-\mathrm{O}(7) & 2.470(2) & \mathrm{Cd}(2)-\mathrm{O}(8) & 2.354(2) \\ \mathrm{Cd}(2)-\mathrm{N}(10) \# 4 & 2.261(2) & & \\ \mathrm{O}(1)-\mathrm{Cd}(1)-\mathrm{O}(2) & 54.44(7) & \mathrm{O}(5)-\mathrm{Cd}(1)-\mathrm{O}(1) & 91.02(7) \\ \mathrm{O}(5)-\mathrm{Cd}(1)-\mathrm{O}(2) & 144.53(8) & \mathrm{O}(5)-\mathrm{Cd}(1)-\mathrm{O}(6) \# 1 & 130.21(8) \\ \mathrm{O}(6) \# 1-\mathrm{Cd}(1)-\mathrm{O}(1) & 138.72(8) & \mathrm{O}(6) \# 1-\mathrm{Cd}(1)-\mathrm{O}(2) & 84.88(8) \\ \mathrm{N}(1)-\mathrm{Cd}(1)-\mathrm{O}(1) & 90.63(8) & \mathrm{N}(1)-\mathrm{Cd}(1)-\mathrm{O}(2) & 100.71(9) \\ \mathrm{N}(1)-\mathrm{Cd}(1)-\mathrm{O}(5) & 85.89(8) & \mathrm{N}(1)-\mathrm{Cd}(1)-\mathrm{O}(6) \# 1 & 90.52(8) \\ \mathrm{N}(1)-\mathrm{Cd}(1)-\mathrm{N}(5) \# 2 & 167.88(8) & \mathrm{N}(5) \# 2-\mathrm{Cd}(1)-\mathrm{O}(1) & 99.09(9) \\ \mathrm{N}(5) \# 2-\mathrm{Cd}(1)-\mathrm{O}(2) & 90.86(9) & \mathrm{N}(5) \# 2-\mathrm{Cd}(1)-\mathrm{O}(5) & 86.72(8)\end{array}$




\begin{tabular}{|c|c|c|c|}
\hline $\mathrm{N}(5) \# 2-\mathrm{Cd}(1)-\mathrm{O}(6) \# 1$ & 86.94(9) & $\mathrm{O}(3) \# 1-\mathrm{Cd}(2)-\mathrm{O}(7)$ & $146.16(9)$ \\
\hline $\mathrm{O}(3) \# 1-\mathrm{Cd}(2)-\mathrm{O}(8)$ & $92.60(9)$ & $\mathrm{O}(4) \# 3-\mathrm{Cd}(2)-\mathrm{O}(3) \# 1$ & $126.27(9)$ \\
\hline $\mathrm{O}(4) \# 3-\mathrm{Cd}(2)-\mathrm{O}(7)$ & $87.31(7)$ & $\mathrm{O}(4) \# 3-\mathrm{Cd}(2)-\mathrm{O}(8)$ & $141.08(7)$ \\
\hline $\mathrm{O}(4) \# 3-\mathrm{Cd}(2)-\mathrm{N}(6)$ & 86.93(9) & $\mathrm{O}(8)-\mathrm{Cd}(2)-\mathrm{O}(7)$ & $53.78(7)$ \\
\hline $\mathrm{N}(6)-\mathrm{Cd}(2)-\mathrm{O}(3) \# 1$ & 84.91(10) & $\mathrm{N}(6)-\mathrm{Cd}(2)-\mathrm{O}(7)$ & $93.82(9)$ \\
\hline $\mathrm{N}(6)-\mathrm{Cd}(2)-\mathrm{O}(8)$ & $95.55(9)$ & $\mathrm{N}(10) \# 4-\mathrm{Cd}(2)-\mathrm{O}(3) \# 1$ & $88.31(10)$ \\
\hline $\mathrm{N}(10) \# 4-\mathrm{Cd}(2)-\mathrm{O}(4) \# 3$ & $94.20(9)$ & $\mathrm{N}(10) \# 4-\mathrm{Cd}(2)-\mathrm{O}(7)$ & $93.83(9)$ \\
\hline $\mathrm{N}(10) \# 4-\mathrm{Cd}(2)-\mathrm{O}(8)$ & $88.36(9)$ & $\mathrm{N}(10) \# 4-\mathrm{Cd}(2)-\mathrm{N}(6)$ & $172.32(9)$ \\
\hline \multicolumn{4}{|l|}{2} \\
\hline $\mathrm{Mn}(1)-\mathrm{O}(5)$ & $2.1490(18)$ & $\mathrm{Mn}(1)-\mathrm{O}(6) \# 5$ & $2.1523(17)$ \\
\hline $\operatorname{Mn}(1)-\mathrm{N}(1)$ & $2.207(2)$ & $\mathrm{Mn}(1)-\mathrm{O}(1)$ & $2.2257(18)$ \\
\hline $\mathrm{Mn}(1)-\mathrm{N}(3) \# 6$ & $2.243(2)$ & $\mathrm{Mn}(1)-\mathrm{O}(2)$ & $2.3210(19)$ \\
\hline $\mathrm{Mn}(2)-\mathrm{O}(3)$ & $2.123(2)$ & $\mathrm{Mn}(2)-\mathrm{O}(4) \# 7$ & $2.1281(19)$ \\
\hline $\mathrm{Mn}(2)-\mathrm{O}(7)$ & $2.1728(18)$ & $\mathrm{Mn}(2)-\mathrm{N}(9) \# 8$ & $2.262(2)$ \\
\hline $\mathrm{Mn}(2)-\mathrm{N}(6)$ & $2.280(2)$ & $\mathrm{Mn}(2)-\mathrm{O}(8)$ & $2.467(2)$ \\
\hline $\mathrm{O}(5)-\mathrm{Mn}(1)-\mathrm{O}(6) \# 5$ & $125.42(7)$ & $\mathrm{O}(5)-\mathrm{Mn}(1)-\mathrm{N}(1)$ & $90.66(8)$ \\
\hline $\mathrm{O}(6) \# 5-\mathrm{Mn}(1)-\mathrm{N}(1)$ & $85.37(8)$ & $\mathrm{O}(5)-\mathrm{Mn}(1)-\mathrm{O}(1)$ & $87.55(7)$ \\
\hline $\mathrm{O}(6) \# 5-\mathrm{Mn}(1)-\mathrm{O}(1)$ & $146.78(7)$ & $\mathrm{N}(1)-\mathrm{Mn}(1)-\mathrm{O}(1)$ & $99.58(8)$ \\
\hline $\mathrm{O}(5)-\mathrm{Mn}(1)-\mathrm{N}(3) \# 6$ & $88.66(8)$ & $\mathrm{O}(6) \# 5-\mathrm{Mn}(1)-\mathrm{N}(3) \# 6$ & $88.22(8)$ \\
\hline $\mathrm{N}(1)-\mathrm{Mn}(1)-\mathrm{N}(3) \# 6$ & $171.62(8)$ & $\mathrm{O}(1)-\mathrm{Mn}(1)-\mathrm{N}(3) \# 6$ & $88.74(8)$ \\
\hline $\mathrm{O}(5)-\mathrm{Mn}(1)-\mathrm{O}(2)$ & $144.95(7)$ & $\mathrm{O}(6) \# 5-\mathrm{Mn}(1)-\mathrm{O}(2)$ & $89.60(7)$ \\
\hline $\mathrm{N}(1)-\mathrm{Mn}(1)-\mathrm{O}(2)$ & $91.06(8)$ & $\mathrm{O}(1)-\mathrm{Mn}(1)-\mathrm{O}(2)$ & $57.67(7)$ \\
\hline $\mathrm{N}(3) \# 6-\mathrm{Mn}(1)-\mathrm{O}(2)$ & $94.30(8)$ & $\mathrm{O}(3)-\mathrm{Mn}(2)-\mathrm{O}(4) \# 7$ & $119.57(8)$ \\
\hline $\mathrm{O}(3)-\mathrm{Mn}(2)-\mathrm{O}(7)$ & $94.79(8)$ & $\mathrm{O}(4) \# 7-\mathrm{Mn}(2)-\mathrm{O}(7)$ & $145.62(8)$ \\
\hline $\mathrm{O}(3)-\mathrm{Mn}(2)-\mathrm{N}(9) \# 8$ & $92.26(9)$ & $\mathrm{O}(4) \# 7-\mathrm{Mn}(2)-\mathrm{N}(9) \# 8$ & $88.66(9)$ \\
\hline $\mathrm{O}(7)-\mathrm{Mn}(2)-\mathrm{N}(9) \# 8$ & $91.05(9)$ & $\mathrm{O}(3)-\mathrm{Mn}(2)-\mathrm{N}(6)$ & $84.95(9)$ \\
\hline $\mathrm{O}(4) \# 7-\mathrm{Mn}(2)-\mathrm{N}(6)$ & $86.21(9)$ & $\mathrm{O}(7)-\mathrm{Mn}(2)-\mathrm{N}(6)$ & $96.66(9)$ \\
\hline $\mathrm{N}(9) \# 8-\mathrm{Mn}(2)-\mathrm{N}(6)$ & $171.98(9)$ & $\mathrm{O}(3)-\mathrm{Mn}(2)-\mathrm{O}(8)$ & $148.40(8)$ \\
\hline $\mathrm{O}(4) \# 7-\mathrm{Mn}(2)-\mathrm{O}(8)$ & $90.45(7)$ & $\mathrm{O}(7)-\mathrm{Mn}(2)-\mathrm{O}(8)$ & $55.61(7)$ \\
\hline $\mathrm{N}(9) \# 8-\mathrm{Mn}(2)-\mathrm{O}(8)$ & $98.48(8)$ & $\mathrm{N}(6)-\mathrm{Mn}(2)-\mathrm{O}(8)$ & $87.72(8)$ \\
\hline \multicolumn{4}{|l|}{3} \\
\hline $\mathrm{Zn}(1)-\mathrm{O}(6) \# 9$ & $1.944(2)$ & $\mathrm{Zn}(1)-\mathrm{O}(1)$ & $1.953(2)$ \\
\hline $\mathrm{Zn}(1)-\mathrm{N}(2) \# 10$ & $2.012(2)$ & $\mathrm{Zn}(1)-\mathrm{N}(1)$ & $2.048(2)$ \\
\hline
\end{tabular}




\begin{tabular}{|c|c|c|c|}
\hline $\mathrm{O}(6) \# 9-\mathrm{Zn}(1)-\mathrm{O}(1)$ & $110.76(9)$ & $\mathrm{O}(6) \# 9-\mathrm{Zn}(1)-\mathrm{N}(2) \# 10$ & $106.45(9)$ \\
\hline $\mathrm{O}(1)-\mathrm{Zn}(1)-\mathrm{N}(2) \# 10$ & $115.36(10)$ & $\mathrm{O}(6) \# 9-\mathrm{Zn}(1)-\mathrm{N}(1)$ & $102.25(9)$ \\
\hline $\mathrm{O}(1)-\mathrm{Zn}(1)-\mathrm{N}(1)$ & $109.84(9)$ & $\mathrm{N}(2) \# 10-\mathrm{Zn}(1)-\mathrm{N}(1)$ & $111.33(10)$ \\
\hline \multicolumn{4}{|l|}{4} \\
\hline $\mathrm{Cu}(1)-\mathrm{N}(4) \# 11$ & $1.971(2)$ & $\mathrm{Cu}(1)-\mathrm{O}(2) \# 12$ & $1.9971(16)$ \\
\hline $\mathrm{Cu}(1)-\mathrm{N}(1)$ & $1.999(2)$ & $\mathrm{Cu}(1)-\mathrm{O}(3) \# 13$ & $2.0349(17)$ \\
\hline $\mathrm{Cu}(1)-\mathrm{O}(1)$ & $2.2286(17)$ & & \\
\hline $\mathrm{N}(4) \# 11-\mathrm{Cu}(1)-\mathrm{O}(2) \# 12$ & $95.21(8)$ & $\mathrm{N}(4) \# 11-\mathrm{Cu}(1)-\mathrm{N}(1)$ & $176.07(8)$ \\
\hline $\mathrm{O}(2) \# 12-\mathrm{Cu}(1)-\mathrm{N}(1)$ & $86.75(8)$ & $\mathrm{N}(4) \# 11-\mathrm{Cu}(1)-\mathrm{O}(3) \# 13$ & $90.91(8)$ \\
\hline $\mathrm{O}(2) \# 12-\mathrm{Cu}(1)-\mathrm{O}(3) \# 13$ & $147.05(8)$ & $\mathrm{N}(1)-\mathrm{Cu}(1)-\mathrm{O}(3) \# 13$ & $89.28(8)$ \\
\hline $\mathrm{N}(4) \# 11-\mathrm{Cu}(1)-\mathrm{O}(1)$ & $89.62(8)$ & $\mathrm{O}(2) \# 12-\mathrm{Cu}(1)-\mathrm{O}(1)$ & $120.75(7)$ \\
\hline $\mathrm{N}(1)-\mathrm{Cu}(1)-\mathrm{O}(1)$ & $86.45(8)$ & $\mathrm{O}(3) \# 13-\mathrm{Cu}(1)-\mathrm{O}(1)$ & $91.57(7)$ \\
\hline \multicolumn{4}{|l|}{5} \\
\hline $\mathrm{Co}(1 \quad)-\mathrm{O}(1)$ & $1.9177(15)$ & $\mathrm{Co}(1)-\mathrm{N}(1)$ & $2.0129(17)$ \\
\hline $\mathrm{Co}(2)-\mathrm{O}(4) \# 15$ & $2.0154(14)$ & $\mathrm{Co}(2)-\mathrm{O}(5)$ & $2.1445(15)$ \\
\hline $\mathrm{Co}(2)-\mathrm{O}(6)$ & $2.1541(14)$ & $\mathrm{Co}(2)-\mathrm{O}(7)$ & $2.1511(18)$ \\
\hline $\mathrm{Co}(2)-\mathrm{O}(8)$ & $2.1541(19)$ & $\mathrm{Co}(2)-\mathrm{N}(3) \# 16$ & $2.0867(17)$ \\
\hline $\mathrm{O}(1)-\mathrm{Co}(1)-\mathrm{O}(1) \# 14$ & $117.97(12)$ & $\mathrm{O}(1) \quad-\mathrm{Co}(1)-\mathrm{N}(1)$ & $97.99(7)$ \\
\hline $\mathrm{O}(1) \# 14-\mathrm{Co}(1)-\mathrm{N}(1)$ & $119.14(8)$ & $\mathrm{N}(1) \# 14-\mathrm{Co}(1)-\mathrm{N}(1)$ & $105.19(10)$ \\
\hline $\mathrm{O}(4) \# 15-\mathrm{Co}(2)-\mathrm{O}(5)$ & $95.49(6)$ & $\mathrm{O}(4) \# 15-\mathrm{Co}(2)-\mathrm{O}(6)$ & $156.67(6)$ \\
\hline $\mathrm{O}(4) \# 15-\mathrm{Co}(2)-\mathrm{O}(7)$ & $85.94(6)$ & $\mathrm{O}(4) \# 15-\mathrm{Co}(2)-\mathrm{O}(8)$ & $89.79(6)$ \\
\hline $\mathrm{O}(4) \# 15-\mathrm{Co}(2)-\mathrm{N}(3) \# 16$ & $99.50(7)$ & $\mathrm{O}(5)-\mathrm{Co}(2)-\mathrm{O}(6)$ & $61.20(5)$ \\
\hline $\mathrm{O}(5) \quad-\mathrm{Co}(2)-\mathrm{O}(7)$ & $89.89(7)$ & $\mathrm{O}(5) \quad-\mathrm{Co}(2)-\mathrm{O}(8)$ & $93.24(8)$ \\
\hline $\mathrm{O}(7)-\mathrm{Co}(2)-\mathrm{O}(6)$ & $92.66(7)$ & $\mathrm{O}(7)-\mathrm{Co}(2)-\mathrm{O}(8)$ & $174.93(6)$ \\
\hline $\mathrm{O}(8)-\mathrm{Co}(2)-\mathrm{O}(6)$ & $92.34(7)$ & $\mathrm{N}(3) \# 16-\mathrm{Co}(2)-\mathrm{O}(5)$ & $164.86(6)$ \\
\hline $\mathrm{N}(3) \# 16-\mathrm{Co}(2)-\mathrm{O}(6)$ & $103.76(6)$ & $\mathrm{N}(3) \# 16-\mathrm{Co}(2)-\mathrm{O}(7)$ & $89.09(7)$ \\
\hline $\mathrm{N}(3) \# 16-\mathrm{Co}(2)-\mathrm{O}(8)$ & $88.92(7)$ & & \\
\hline \multicolumn{4}{|l|}{6} \\
\hline $\mathrm{Co}(1)-\mathrm{N}(3) \# 17$ & $2.082(3)$ & $\mathrm{Co}(1)-\mathrm{O}(7)$ & $2.127(3)$ \\
\hline $\mathrm{Co}(1)-\mathrm{O}(1)$ & $2.158(2)$ & $\mathrm{Co}(2)-\mathrm{O}(2) \# 4$ & $1.969(2)$ \\
\hline $\mathrm{Co}(2)-\mathrm{O}(3)$ & $1.983(2)$ & $\mathrm{Co}(2)-\mathrm{O}(5) \# 5$ & $2.004(2)$ \\
\hline $\mathrm{Co}(2)-\mathrm{N}(1)$ & $2.012(3)$ & & \\
\hline
\end{tabular}




$\begin{array}{llll}\mathrm{N}(3) \# 17-\mathrm{Co}(1)-\mathrm{N}(3) \# 18 & 180.000(1) & \mathrm{N}(3) \# 17-\mathrm{Co}(1)-\mathrm{O}(7) & 87.70(11) \\ \mathrm{N}(3) \# 18-\mathrm{Co}(1)-\mathrm{O}(7) & 92.30(11) & \mathrm{O}(7)-\mathrm{Co}(1)-\mathrm{O}(7) \# 19 & 180.0 \\ \mathrm{~N}(3) \# 18-\mathrm{Co}(1)-\mathrm{O}(1) & 95.07(9) & \mathrm{N}(3) \# 17-\mathrm{Co}(1)-\mathrm{O}(1) & 84.93(9) \\ \mathrm{O}(7)-\mathrm{Co}(1)-\mathrm{O}(1) \# 19 & 92.05(11) & \mathrm{O}(7)-\mathrm{Co}(1)-\mathrm{O}(1) & 87.95(11) \\ \mathrm{O}(1) \# 19-\mathrm{Co}(1)-\mathrm{O}(1) & 180.000(1) & \mathrm{O}(2) \# 20-\mathrm{Co}(2)-\mathrm{O}(3) & 108.33(10) \\ \mathrm{O}(2) \# 20-\mathrm{Co}(2)-\mathrm{O}(5) \# 21 & 98.41(9) & \mathrm{O}(3)-\mathrm{Co}(2)-\mathrm{O}(5) \# 21 & 104.32(11) \\ \mathrm{O}(2) \# 20-\mathrm{Co}(2)-\mathrm{N}(1) & 110.18(10) & \mathrm{O}(3)-\mathrm{Co}(2)-\mathrm{N}(1) & 118.27(11) \\ \mathrm{O}(5) \# 21-\mathrm{Co}(2)-\mathrm{N}(1) & 115.33(10) & & \end{array}$

symmetry codes: \#1 - $x, 1-y, 2-z, \# 2-1+x,-1+y, z, \# 3 \quad 1+x, y, 1+z, \# 4 x, 1+y, z, \# 5$ $-x+1,-y+1,-z+1, \# 6 x, y+1, z, \# 7-x+2,-y+1,-z, \# 8 x-1, y+1, z, \# 9 x-1 / 2,-y+1 / 2, z-1 / 2, \# 10$ $-x+1 / 2, y-1 / 2,-z-1 / 2, \# 11 x+1, y, z-1, \# 12-x+1,-y+1,-z+1, \# 13-x+1,-y+2,-z+1,14 \#$ $-x,+y, 1 / 2-x, \quad 15 \# \quad x, 1-y,-1 / 2+z, \quad 16 \# \quad 1 / 2+x,-3 / 2+y,+z, \quad 17 \# \quad x-1, y-1, z+1, \quad 18 \#$ $-x+2,-y+1,-z+1,19 \#-x+1,-y,-z+2,20 \#-x+1,-y+1,-z+2,21 \# x+1, y, z$.

(a)

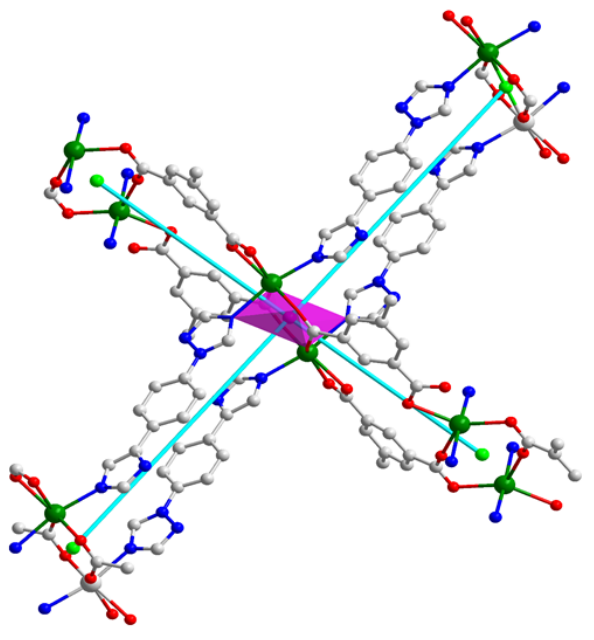

(b)

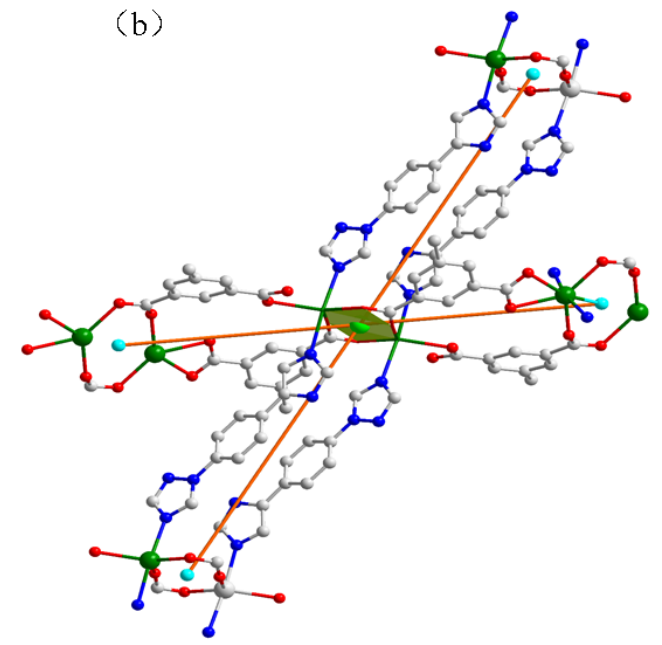

Figure S1 Ball-and-stick representations of two kinds of 4-connected Cd(II) atoms in MOF 1. 


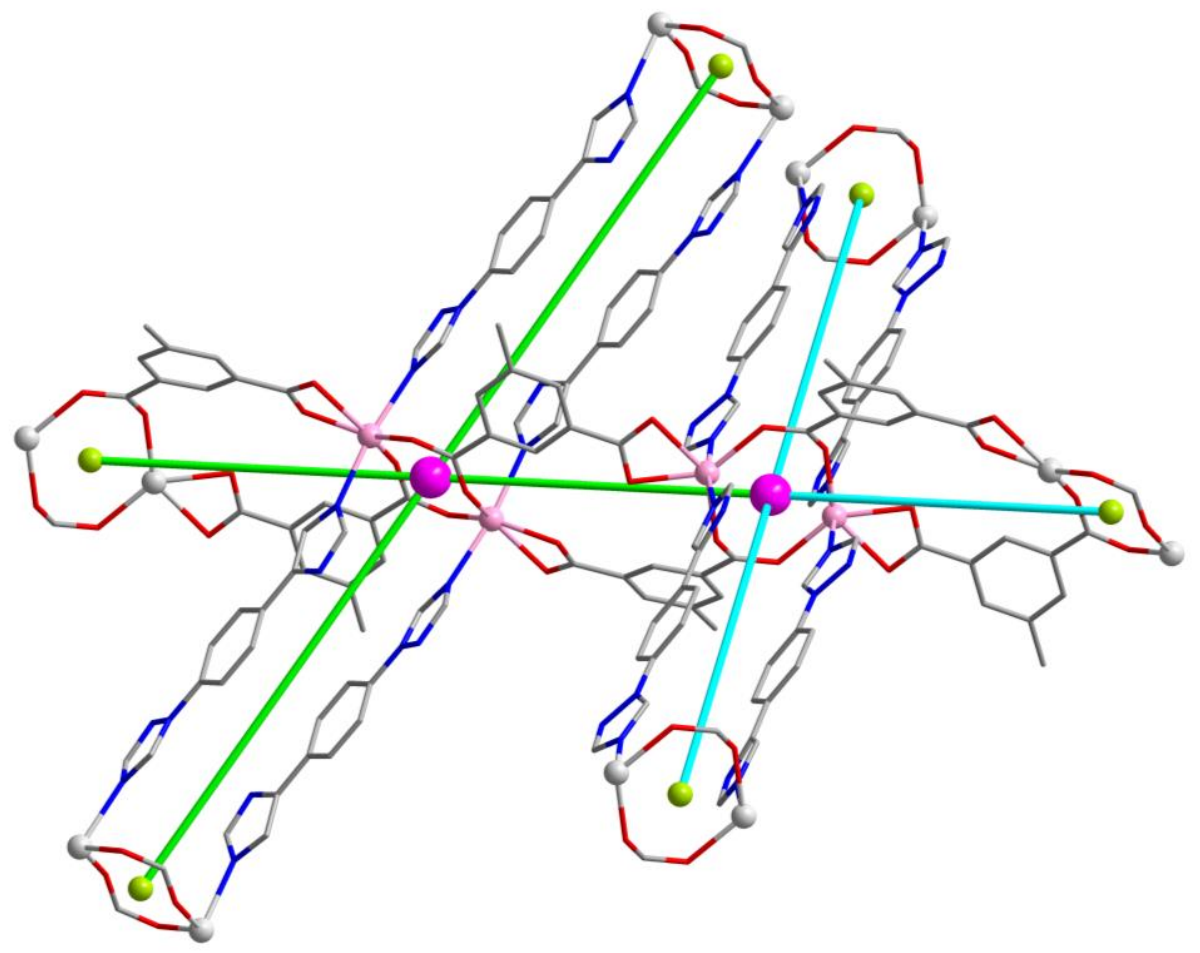

Figure S2 Ball-and-stick representations of two kinds of 4-connected binuclear Cd(II) cluster of 2.

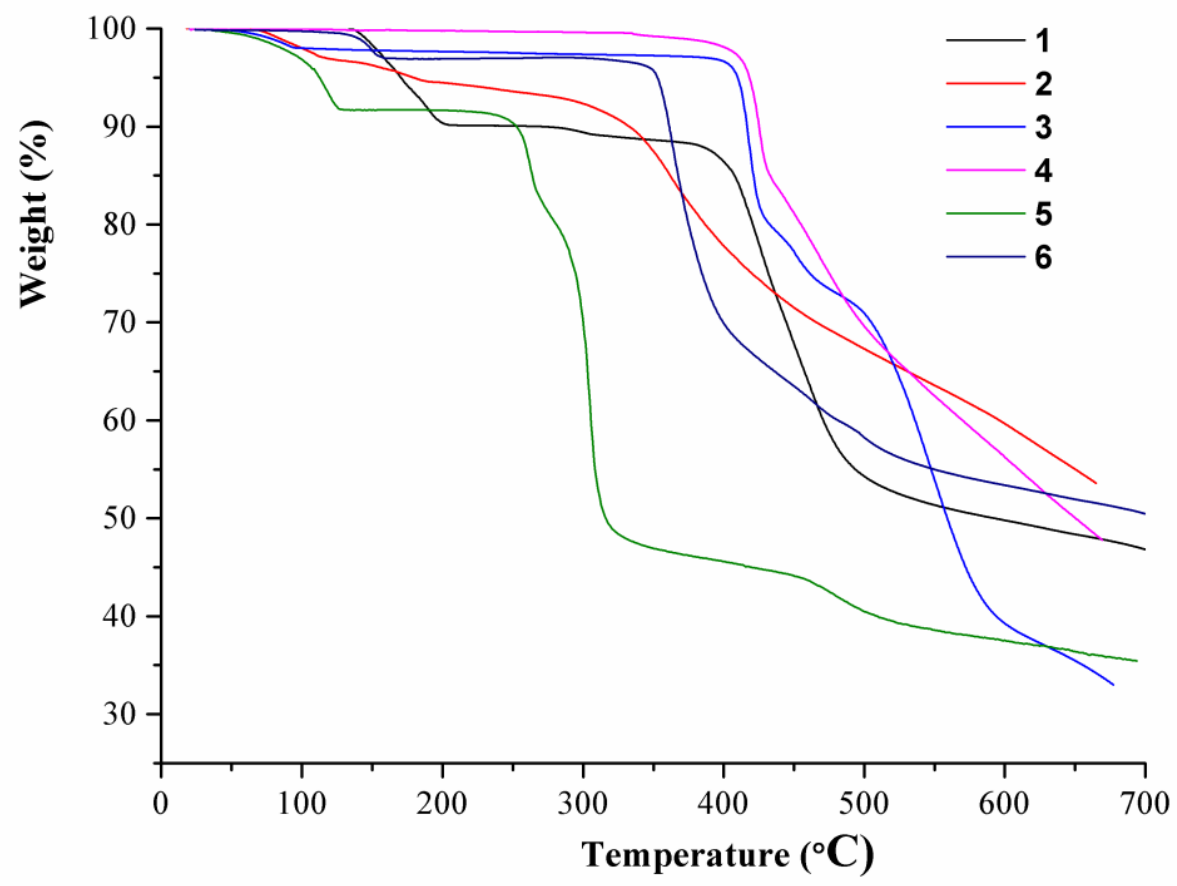

Figure S3 TG curves of MOFs 1 - 6. 

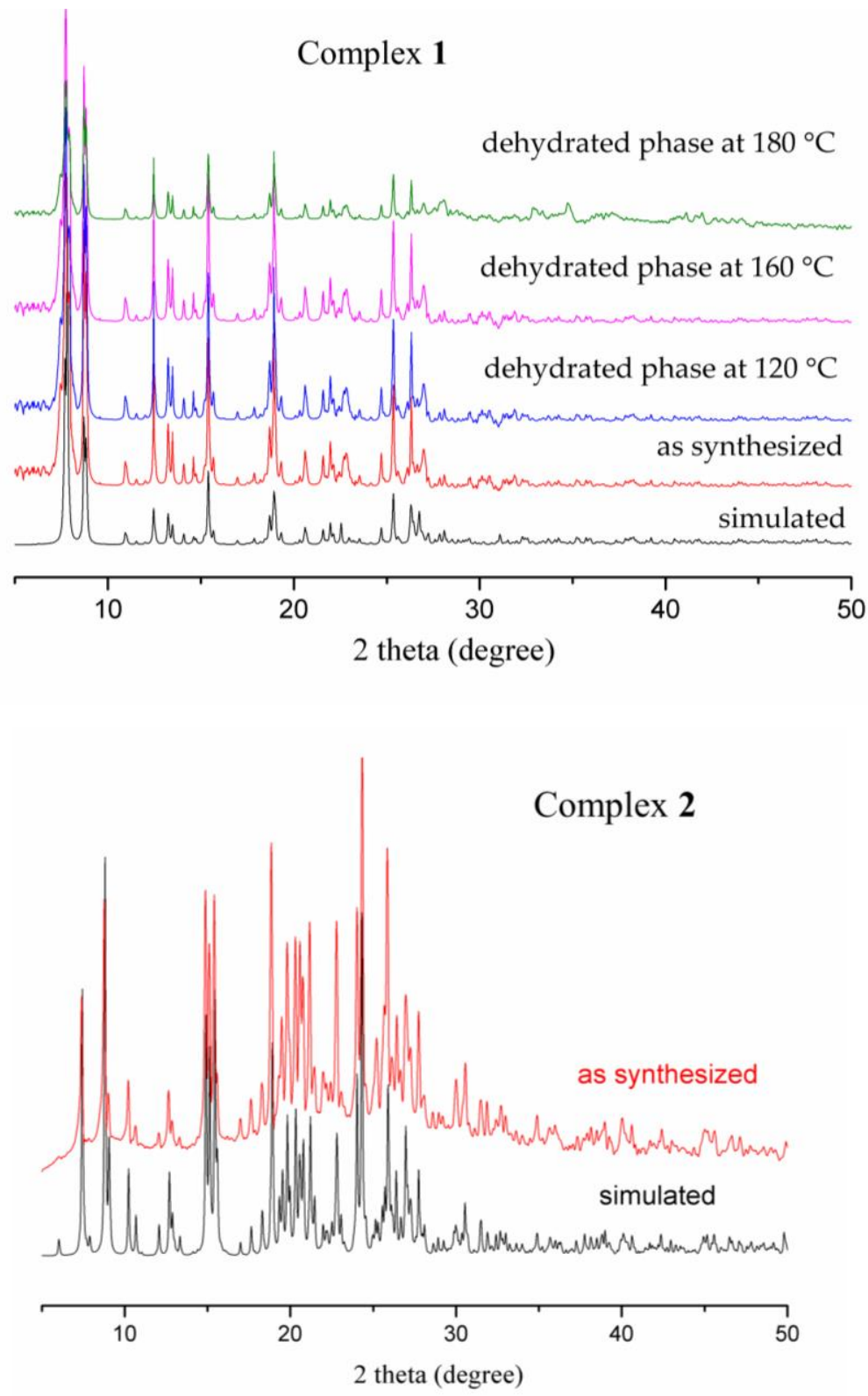

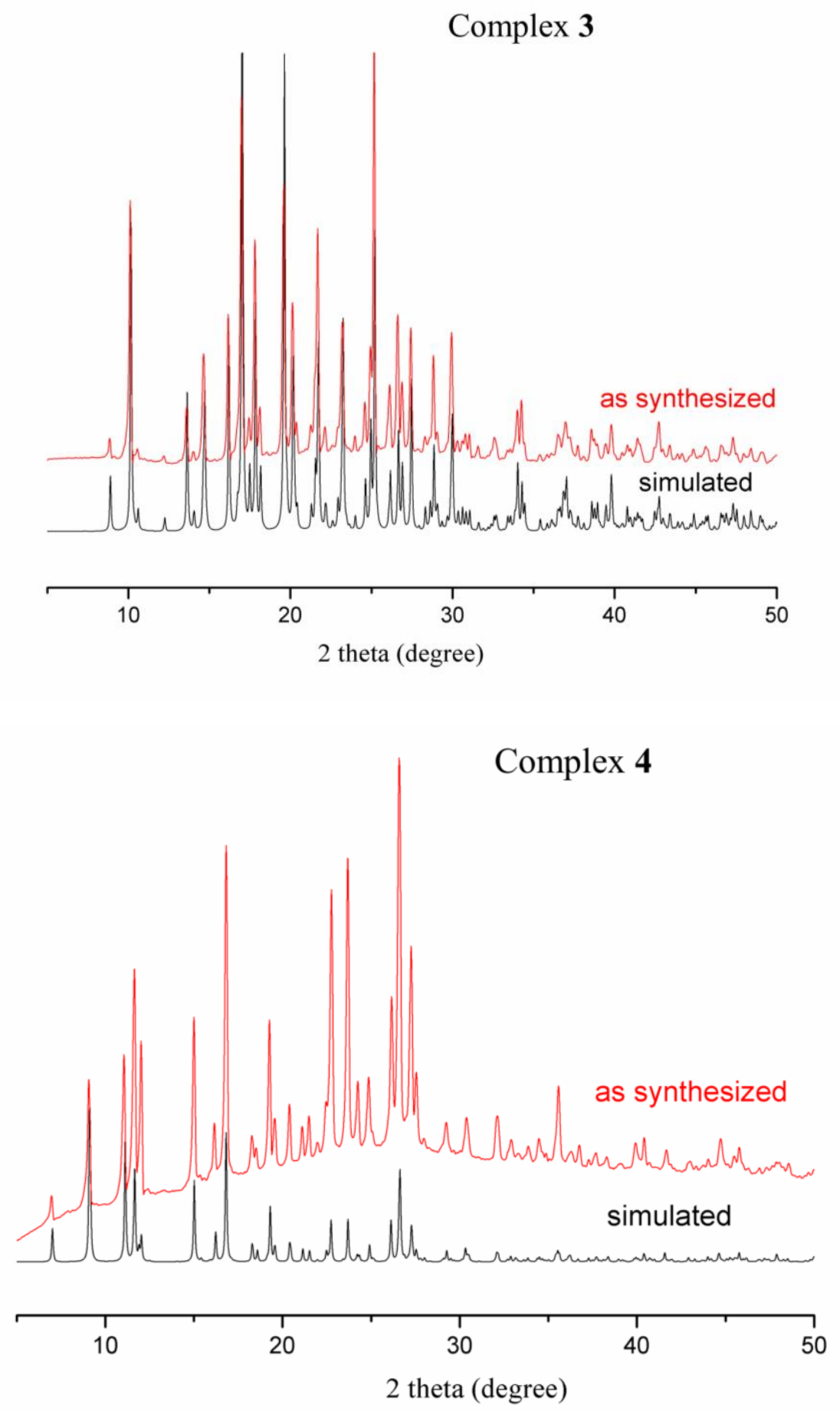

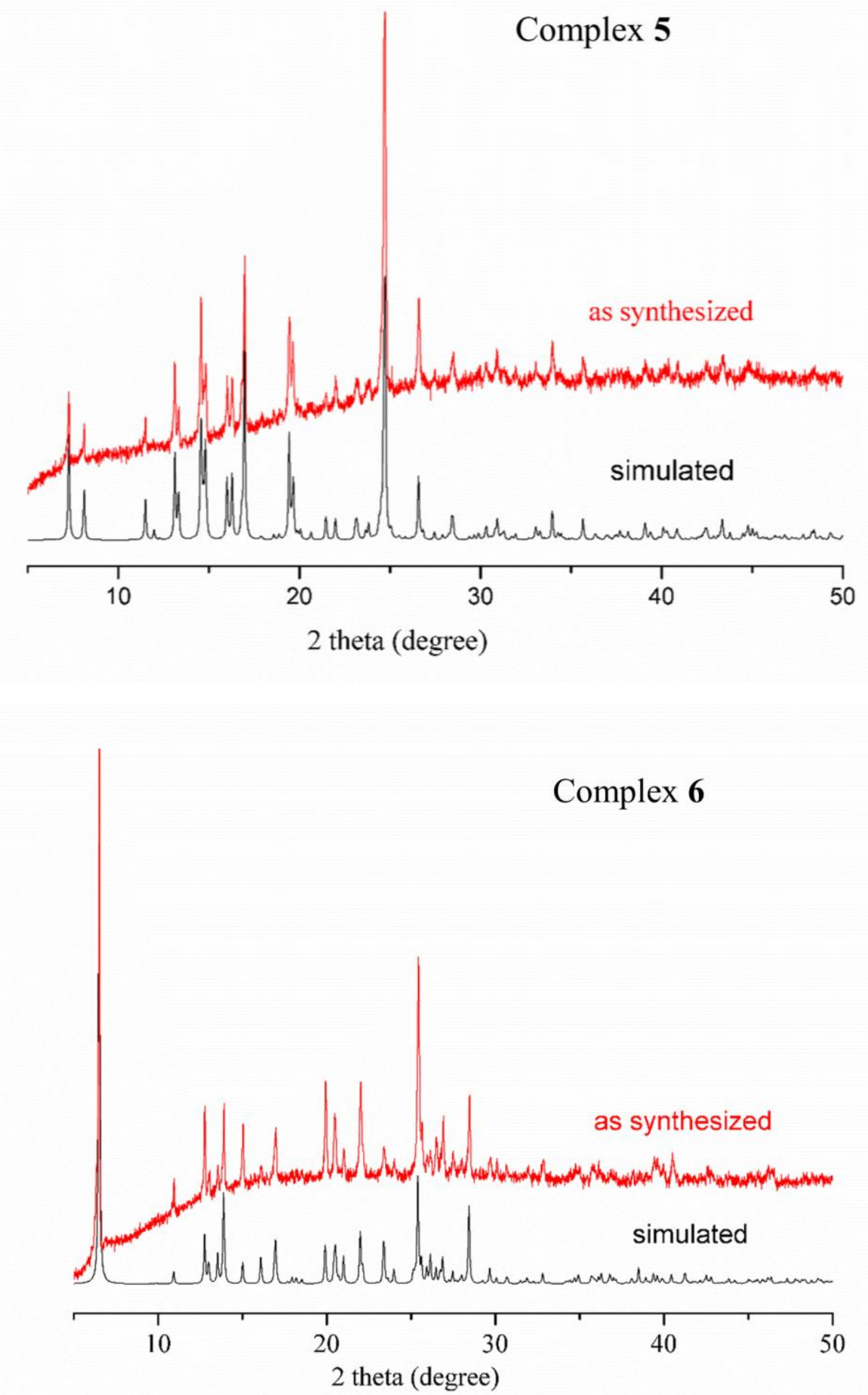

Figure S4 X-ray powder diffraction patterns of MOFs $\mathbf{1}-\mathbf{6}$. 

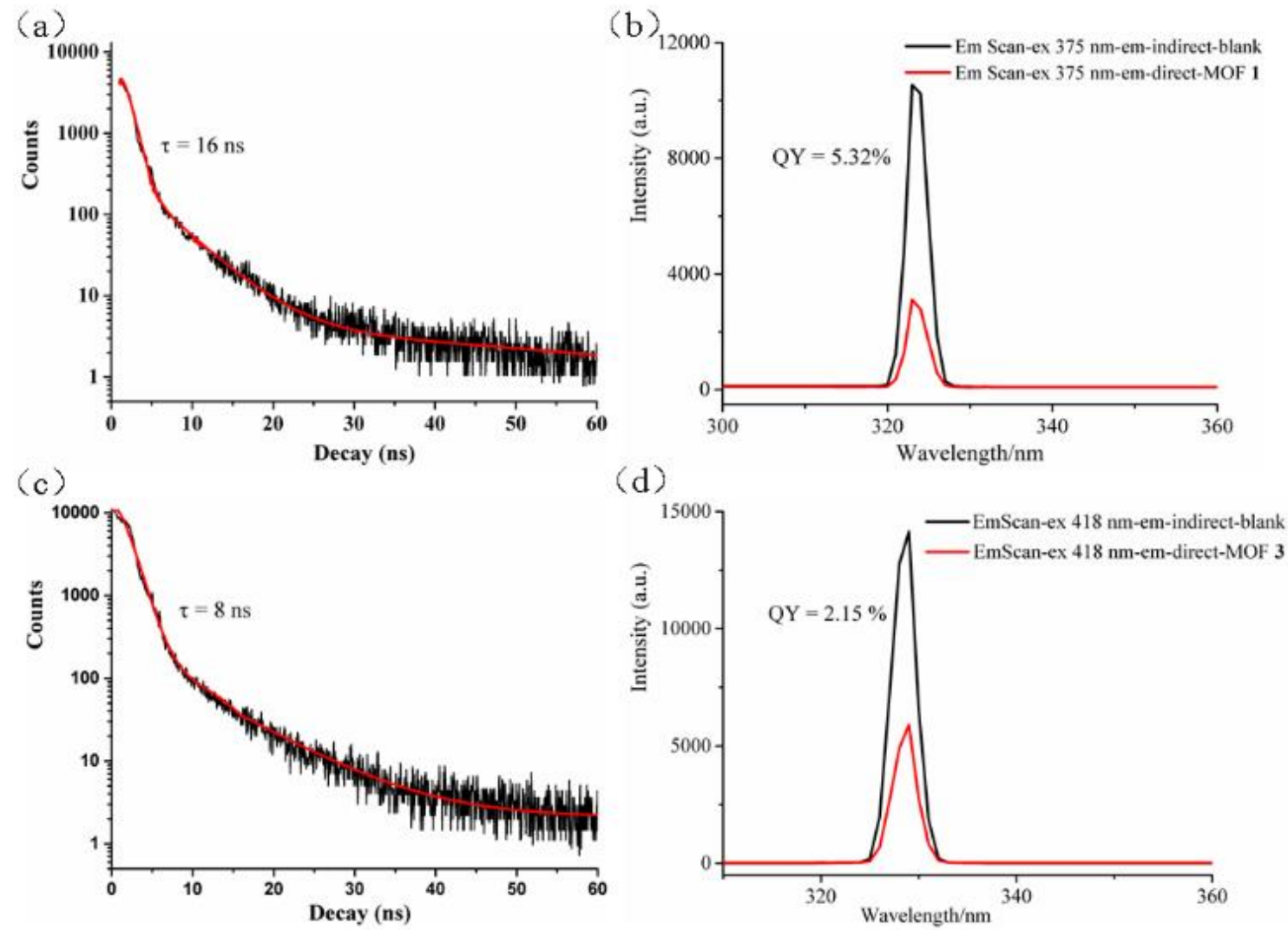

Figure S5. The decay curves and QY of MOFs 1 and 3.

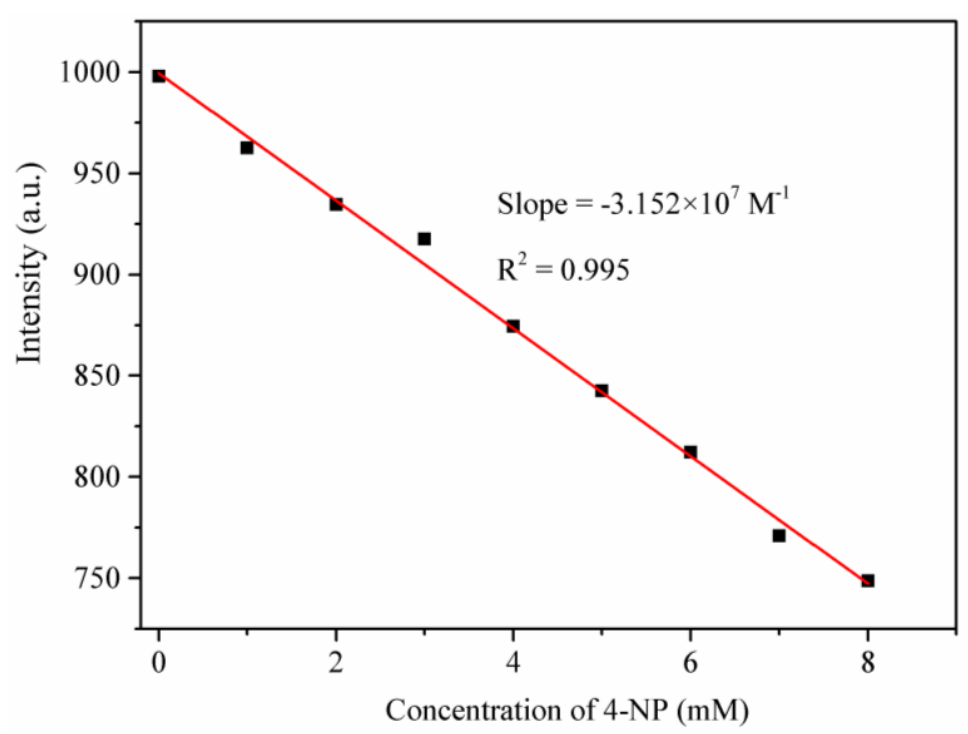

Linear Equation: $\mathrm{Y}=-31.52 \mathrm{x}+999.58 \quad \mathrm{R}^{2}=0.995$

$$
\begin{gathered}
\text { Slope }=3.152 \times 10^{7} \mathrm{M}^{-1} \quad \delta=3.453(\mathrm{~N}=10) \\
\text { Limit detection }=3 \delta / \text { Slope }=3.29 \times 10^{-7}
\end{gathered}
$$

Figure S6 The fitting curve of the luminescence intensity of 1 at different 4-NP concentration. 

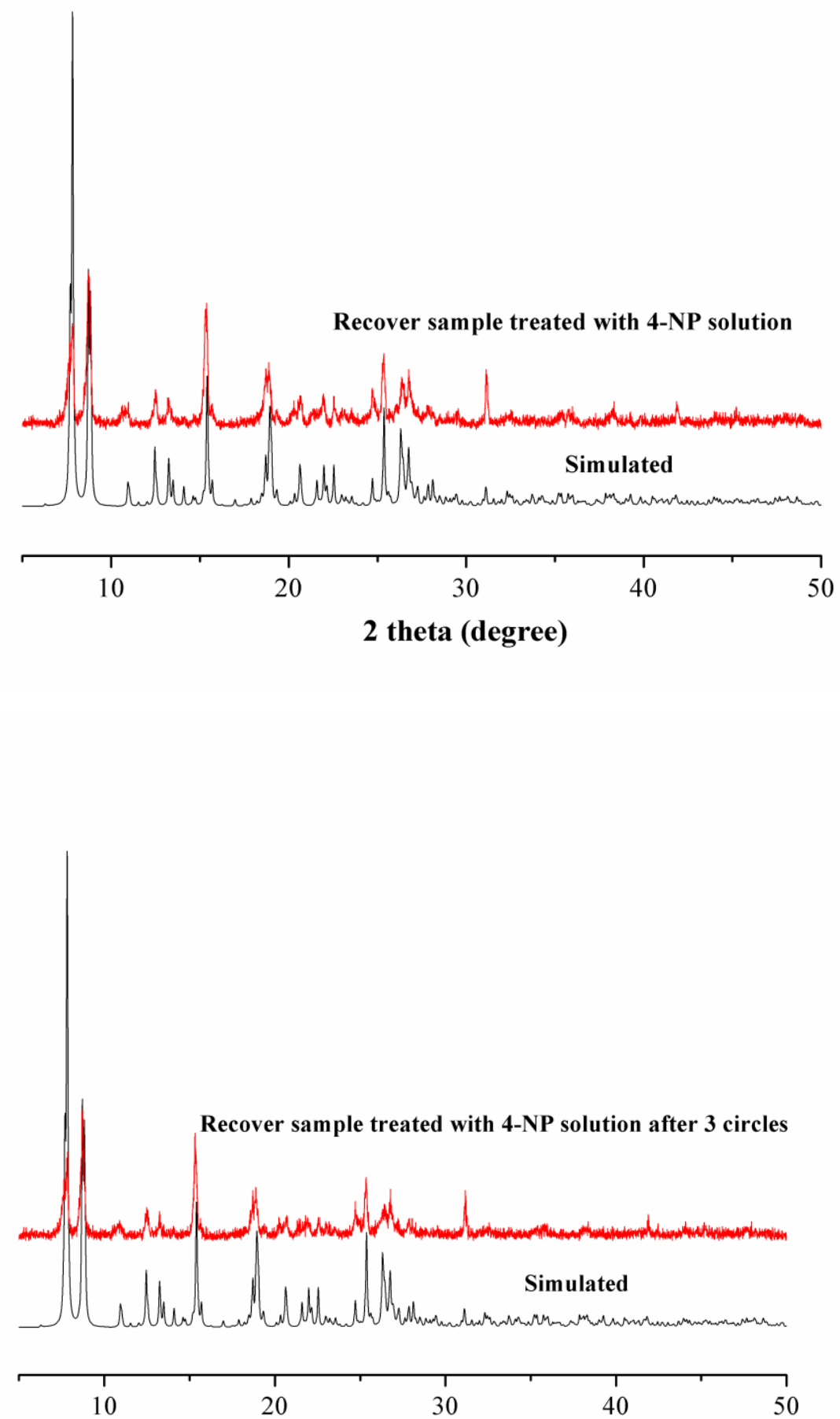

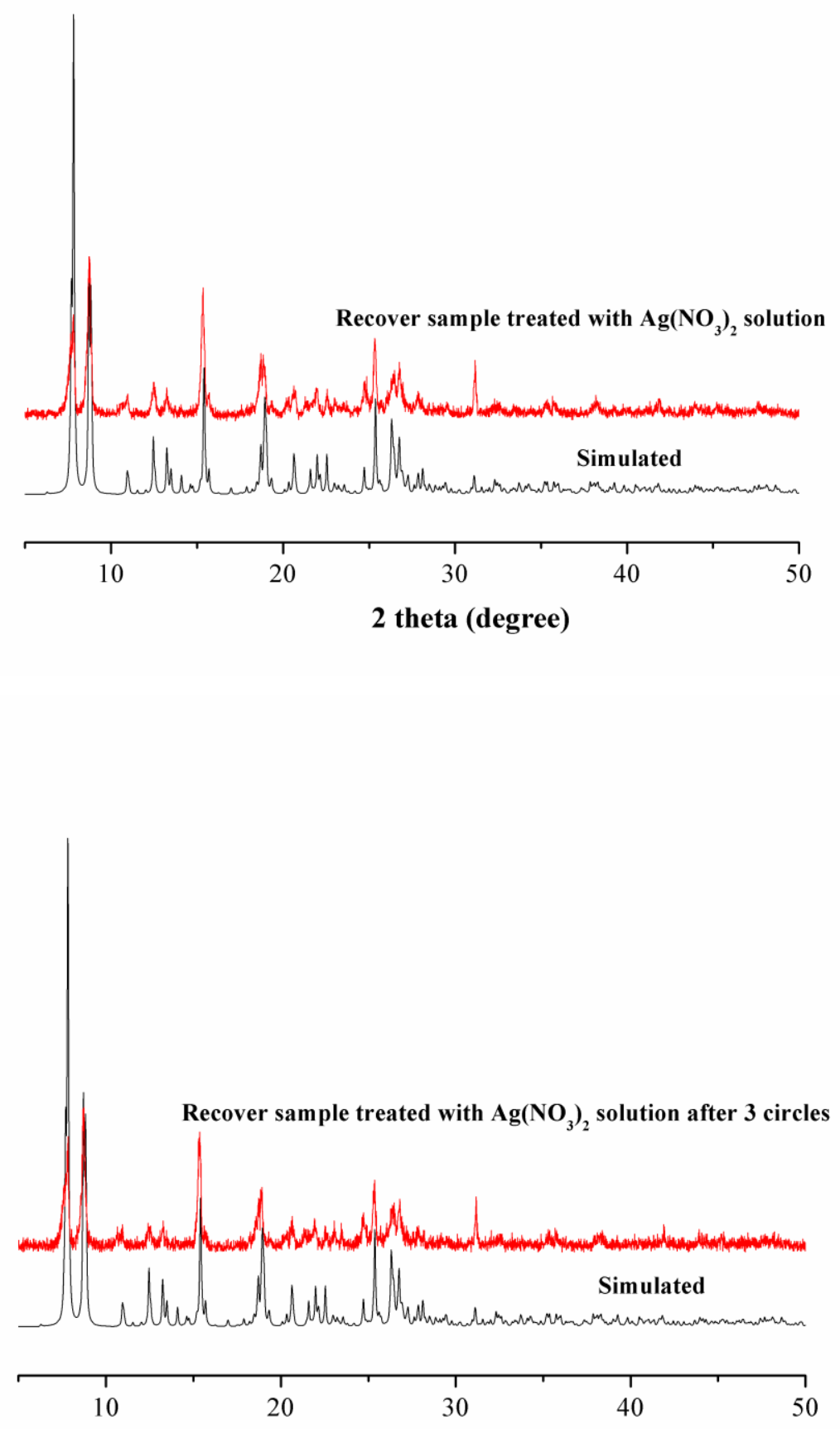

Figure S7 X-ray powder diffraction patterns treated with guest molecules solution for MOF 1. 


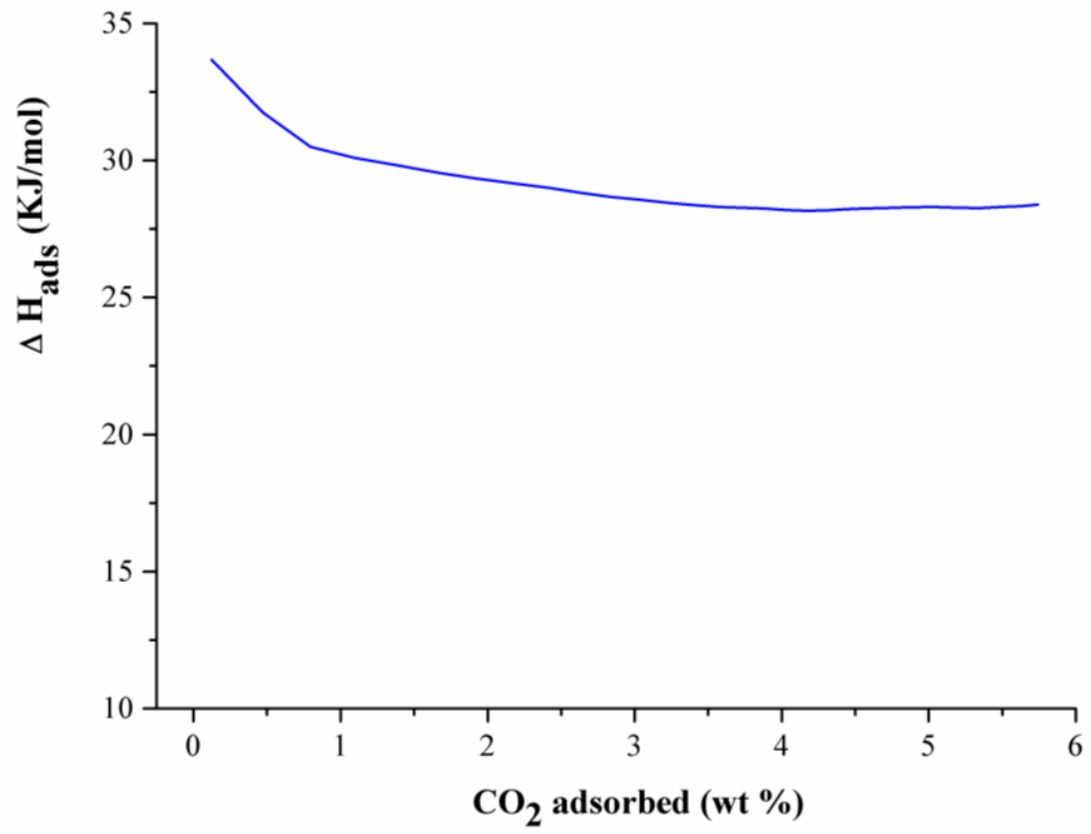

Figure S8. $\mathrm{CO}_{2}$ adsorption enthalpy for $\mathbf{1}^{\prime}$ calculated from the $\mathrm{CO}_{2}$ adsorption isotherms at $273 \mathrm{~K}$ and $298 \mathrm{~K}$. 